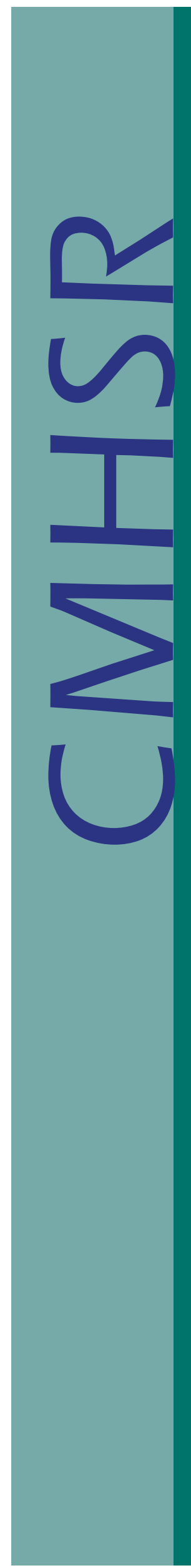

Nov 2004

Vol 1, Issue 9

\title{
Assertive Community Treatment: Re-thinking a Tried and Tested Service Model
}

Robert King, PhD, FAPS. School of Medicine, The University of Queensland and Visiting Scholar, Program for Clubhouse Research, Center for Mental Health Services Research, The University of Massachusetts Medical School

\section{A} ssertive Community Treatment (ACT) is a team-based, mobile, high contact model of mental health service delivery for people with disability and high risk of relapse. Typical interventions by ACT teams include delivery of medications, monitoring mental state, intensive work on everyday living skills, response to crises, assistance with stable accommodations, and facilitation of access to other community supports and clinical services. Case managers in standard care perform broadly similar roles but at much lower levels of intensity and with higher caseloads. Standard case management relies more on the initiative and active collaboration of the client whereas ACT as the name implies, empowers the clinician to engage clients who are ambivalent about or even resistant to services.

The early success of ACT, especially in reducing the need for frequent re-admission to hospital was well researched and documented, resulting in widespread dissemination and implementation of the model as an evidence-based treatment program. ${ }^{1}$ Meta-analyses of studies that have compared ACT with standard care have indicated that ACT is associated with reduced hospital use, high consumer satisfaction and probably with improved social and clinical outcomes. ACT has been recognized, as an evidence-based service response to severe mental illness, by public health authorities in the US. The model has had the enthusiastic support both of influential advocacy groups and the Substance Abuse and Mental Health Services Administration (SAMHSA).

(C) 2004 Center for Mental Health Services Research Department of Psychiatry

University of Massachusetts Medical School

\section{Shortcomings of ACT}

While the ACT model has played an important role in the transition from institutional to community-based care of people with major mental illness, it does have some shortcomings and it is time to reconsider its role in contemporary mental health services. The shortcomings may be summarized as follows:

- $\quad$ ACT is an expensive model of communitybased treatment, largely due to a low staffclient ratio (usually 1:8-10)

- ACT is probably only cost-effective if clients would otherwise receive 50 or more days each year of inpatient treatment ${ }^{2}-$ while extended periods of inpatient treatment were not unusual when ACT was introduced, current admission and discharge practices make cost effectiveness more difficult to achieve.

- Recent evidence ${ }^{3,4,5}$ suggests that ACT may not result in substantially better clinical or social client outcomes than standard care. This is probably because standard care is more effective than was the case when ACT was first introduced, partly because some ACT practices have been introduced to standard care.

- ACT is more coercive than standard care in that it clients are often subjected to psychological pressure to comply with medication regimes and to accept home visits and sometimes other interventions. It tends to operate with in a traditional medical and disability model rather than the more contemporary recovery and strengths model.

Proponents of ACT have acknowledged some of these shortcomings and have attempted to address them.5,6,7 Strengthening and updating the 
model has taken two forms; 1.) focus on fidelity in delivery and 2.) strengthening the standards to reflect current recovery focus.

\section{Strengthening ACT through increased fidelity}

Focusing on fidelity is designed to ensure that ACT is delivered in accordance with published standards. However, it is by no means clear that having a fidelity focus will solve the problems that ACT is facing. First, it is not clear which components of ACT are vital to its success. The published standards do not have a strong empirical foundation and greater enforcement of standards may not improve service outcomes. One major study that reported no impact of ACT, compared with usual care, found that fidelity was acceptable. ${ }^{8}$ Second, high fidelity may be difficult to sustain in real-world service environments. A program that initially meets certification standards may fall below them at various times because of availability of staff or changes in the funding environment. A model that is dependent on sustained high fidelity may not be robust enough for routine application in clinical environments. ${ }^{9}$

\section{Strengthening ACT standards through recovery focus}

Building a recovery focus might seem to be straightforward, especially when there is evidence that focus on strengths is both possible and leads to better outcomes. ${ }^{10}$ However, there is a strong tradition, within the ACT model, of illness focus and assertive provision of medication. The evidence suggests that, even in the face of evidence that focus on social integration enhances outcomes, the large majority of ACT teams maintain a clinical focus 11 and non-clinical team members are the exception rather than the rule.

\section{The future of ACT}

It is possible that the longer-term legacy of ACT will not be its success as a specific program but rather its influence on the way that usual care is delivered in the community mental health environment. There is evidence that usual care has evolved from an office-based service modeled on the outpatient clinic to a more flexible, mobile and responsive case management that can increase intensity of contact according to need. ${ }^{8}$ It is also relevant in considering the future of ACT to look at changes in the wider service environment that have occurred since the introduction of the ACT model. There are now many more non-government services with a rehabilitation or disability support focus for people with major mental illness. In practice the non-government service environment in the US varies in distribution and quality. However, decisions concerning the initiation or continuation of ACT teams should take into account the characteristics of the community and its services and not just the characteristics of clients.

Future research should give consideration to the development of a stronger evidence base for fidelity components, to more sophisticated modeling of costbenefit and to the investigation of recovery and strengths-based service delivery approaches.

\section{References}

1. Mueser, K.T., Bond, G.R., Drake, R.E., \& Resnick, S.G. (1998). Models of community care for severe mental illness: A review of research on case management. Schizophrenia Bulletin., 24 (1), 37-74.

2. Latimer, E.A. (1999). Economic impacts of assertive community treatment: A review of the literature. Canadian Journal of Psychiatry, 44, 443-54.

3. Burns,T., Creed, F., Fahy, T., et al. (1999). Intensive versus standard case management for severe psychotic illness trial. UK700 Group. Lancet, 353, 21852189.

4. Issakidis, C., et al. (1999). Intensive case management in Australia: A randomized controlled trial. Acta Psychiatrica Scandinavica, 99, 360-7.

5. Clarke, G.N., et al. (2000). Psychiatric hospitalizations, arrests, emergency room visits, and homelessness of clients with serious and persistent mental illness: Findings from a randomized trial of two ACT programs vs. usual care. Mental Health Services Research, 2, 155-64.

6. Marshall, M., \& Creed, F. (2000) .Assertive community treatment - is it the future of community care in the UK? International Review of Psychiatry, 12, 191-196.

7. Allness, D., \& Knoedler, W. (2003). National program standards for ACT teams (2003 revision). Arlington, VA: NAMI; http://www.nami.org/Content/Content Groups/Programs/PACT1/NationalStandardsforACTeams.pdf

8. Fiander, M., Burns, T., McHugo, G.J., \& Drake, R.E. (2003). Assertive community treatment across the Atlantic: Comparison of model fidelity in the UK and USA. British Journal of Psychiatry, 182, 248-54.

9. Duan, N., Braslow, J., Weisz, J., \& Wells K (2001). Fidelity, adherence, and robustness of interventions. Psychiatric Services, 52, 413.

10. Barry, K.L., Zeber, J.E., Blow, F.C., \& Valenstein, M. (2003) . Effect of strengths model versus assertive community treatment model on participant outcomes and utilization: Two-year follow-up. Psychiatric Rehabilitation Journal, 26 (3), 268-77.

11. Salyers, M.P., et.al. (2003). Is it ACT yet? Real-world examples of evaluating the degree of implementation for assertive community treatment. Journal of Behavioral Health Services Research, 30, 304-20.

Visit us on-line at www.umassmed.edu/cmhsr 